\title{
Effect of porcine circovirus type 2 (PCV2) vaccination on PCV2-viremic piglets after experimental PCV2 challenge
}

\author{
Hwi Won Seo, Changhoon Park, Kiwon Han and Chanhee Chae*
}

\begin{abstract}
The objective of this study was to evaluate the effect of porcine circovirus type 2 (PCV2) vaccines on PCV2-viremic and -seropositive piglets born from naturally PCV2-infected sows against postnatal PCV2 challenge. The experimental design was aimed at mimicking commercial swine rearing conditions to evaluate the response of the PCV2 vaccine on PCV2-viremic and -seropositive piglets after experimental PCV2 challenge. PCV2a (or 2b)-viremic piglets received a PCV2 vaccine at 21 days of age followed by a PCV2b (or 2a) challenge at 49 days of age (28 days post vaccination). The PCV2 vaccines elicited a high level of humoral (as measured by immunoperoxidase monolayer assay and neutralizing antibody titers) and cellular (as measured by the frequency of PCV2-specific interferon- $\gamma$-secreting cells) immune response in the PCV2-viremic piglets after vaccination even in the presence of maternally derived antibodies (MDA). The initial infection of PCV2 in the pigs was not affected by PCV2 vaccination, however the challenging PCV2 was reduced by PCV2 vaccination on PCV2-viremic pigs. The results from this study demonstrate that the PCV2 vaccine used in this study is effective at reducing PCV2 viremia and lymphoid PCV2 DNA, even for PCV2-viremic pigs with passively acquired MDA at the time of vaccination.
\end{abstract}

\section{Introduction}

Porcine circovirus type 2 (PCV2), a small, non-enveloped, single stranded circular DNA virus belonging to the genus Circovirus of the family Circoviridae [1], is the causative agent of several diseases and syndromes, collectively referred to as porcine circovirus-associated disease (PCVAD) [2]. Among these conditions, postweaning multisystemic wasting syndrome (PMWS) is the most important [3]. PCVAD has now been recognized worldwide and is considered to be an economically important global disease. Today, most farms use a PCV2 vaccine for the control of PCVAD since the first introduction of the PCV2 vaccine in 2006 [4].

Epidemiologic surveys have reported that $33-40 \%$ of newborn and pre-suckle piglets are PCV2-viremic [5,6]. The PCV2-viremic piglets from PCV2-infected sows were able to develop PMWS during the postnatal period if they were postnatally infected with porcine parvovirus [7]. In addition, piglets from PCV2-viremic sows were at high risk for developing PCVAD at any time throughout

\footnotetext{
* Correspondence: swine@snu.ac.kr
Department of Veterinary Pathology, College of Veterinary Medicine, Seoul

* Correspondence: swine@snu.ac.kr
Department of Veterinary Pathology, College of Veterinary Medicine, Seoul National University, 1 Gwanak-ro, Gwanak-gu, Seoul 151-742, Republic of Korea
}

\section{() Biomed Central}

(c) 2014 Seo et al.; licensee BioMed Central Ltd. This is an open access article distributed under the terms of the Creative Commons Attribution License (http://creativecommons.org/licenses/by/2.0), which permits unrestricted use, distribution, and reproduction in any medium, provided the original work is properly cited. their life [8]. These results suggest that PCV2-viremic piglets may be immune-compromised, resulting in impaired PCV2 immunizations at postnatal periods. Consequently, immune-compromised PCV2-viremic piglets may be one of the contributing factors for increasing reports of apparent vaccine failure in late finisher pigs.

The vaccination of sows has been shown to reduce the prevalence of PCV2 viremia in their piglets under field conditions [9]. Additionally, vaccination of the sow was able to protect piglets against a PCV2 challenge up to 8 weeks of age [10]. This may explain why piglet vaccination is likely to become more popular among pig producers worldwide [4]. The results from one experimental study indicated that PCV2 vaccines can be efficacious in PCV2viremic pigs against triple challenged with $\mathrm{PCV} 2$, porcine reproductive and respiratory syndrome virus (PRRSV) and porcine parvovirus (PPV) [11]. However, this study is focused on the evaluation of the efficacy of the PCV2 vaccine against triple challenge rather than the effect of PCV2 vaccination on PCV2-viremic pigs. Hence, this study aimed to evaluate the effect of PCV2 vaccines on PCV2-viremic pigs from naturally PCV2-infected sows against postnatal PCV2 
challenge. To conduct this experiment, the vaccinated PCV2a (or 2b)-viremic pigs were challenged with PCV2b (or 2a) to differentiate initial PCV2 infection from challenging virus.

\section{Materials and methods}

\section{Animals}

A total of 105 colostrum-fed, cross-bred, conventional piglets were purchased at 7 days of age, derived from 20 sows on a commercial farm. Gilts and sows were not vaccinated for PCV2 on this farm. Clinical signs indicative of PCVAD had not been observed on the farm. Upon arrival, all pigs were tested and found to be negative for PRRSV and Mycoplasma hyopneumoniae according to routine serological testing.

Among the 105 pigs, 84 pigs were PCV2 seropositive and either PCV2a- or PCV2b-viremic. All PCV2-viremic pigs were the same PCV2 type as their dam. PCV2viremic and -seropositive piglets had positive immunoperoxidase monolayer assay (IPMA) titers (ranging from 8 to $12 \log _{2}$ ) for the detection of total PCV2 antibodies, neutralizing antibody (NA) titers (ranging from 7.0 to $9.0 \log _{2}$ ), and genomic copies of PCV2 DNA load in the blood (ranging from 3.5 to $4.5 \log _{10}$ PCV2 DNA copies/ $\mathrm{mL}$ ). The PCV2-viremic and -seropositive pigs used in this study had similar PCV2 viremia and serological profiles to naturally infected piglets (mean $\log _{10}$ PCV2 DNA copies ranging from 3.75 to 4.58 [5] and mean $\log _{2}$ IPMA titers ranging from 10 to 12 [12]). Twenty-one pigs were non-PCV2-viremic and seronegative for PCV2. The pigs were blocked into PCV2a, PCV2b and negative groups prior to randomization and housed separately within the facility in an environmentally controlled building as previously described [13].

\section{PCV2 vaccines}

Commercial and experimental PCV2 vaccines were used in this study. The commercial PCV2 vaccine is an inactivated chimeric PCV1-2 vaccine (Fostera PCV, Zoetis, Madison, NJ, USA). The experimental inactivated PCV2 vaccine contained inactivated PCV2b (at a titer of $10^{6}$ fluorescent antibody infectious dose $50 / \mathrm{mL}$ ) and an aluminum hydroxide gel adjuvant ( $10 \%$ of volume in $1 \mathrm{~mL} /$ dose). PCV2 vaccines were used and administered according to the manufacturer's instructions with regards to time and route of injection (intramuscularly in the right side of the neck).

\section{Experimental design}

The experimental design is summarized in Table 1. A total of 105 pigs were randomly divided into 15 groups (7 pigs per group). Two groups of PCV2a-viremic pigs (groups 1 and 3) and PCV2b-viremic pigs (groups 7 and 9) were immunized with an inactivated chimeric PCV12 vaccine administered as a $2.0 \mathrm{~mL}$ dose at 21 days of age based on the manufacturer's recommendations. Another 2 groups of PCV2a-viremic pigs (groups 2 and 4) and PCV2b-viremic pigs (groups 8 and 10) were

Table 1 Study design with exposed, vaccination, and challenge statuses for PCV2 at different days post challenge (dpc) ${ }^{\mathrm{a}}$

\begin{tabular}{|c|c|c|c|c|c|c|}
\hline \multirow[t]{2}{*}{ Group } & \multicolumn{2}{|c|}{ Viremia (-42 dpc) } & \multicolumn{2}{|c|}{ Vaccination (-28 dpc) } & \multicolumn{2}{|c|}{ Challenge (0 dpc) } \\
\hline & PCV2a & PCV2b & Vaccine $A^{b}$ & Vaccine $B^{c}$ & PCV2a & PCV2b \\
\hline 1 & + & - & + & - & - & + \\
\hline 2 & + & - & - & + & - & + \\
\hline 3 & + & - & + & - & - & - \\
\hline 4 & + & - & - & + & - & - \\
\hline 5 & + & - & - & - & - & + \\
\hline 6 & + & - & - & - & - & - \\
\hline 7 & - & + & + & - & + & - \\
\hline 8 & - & + & - & + & + & - \\
\hline 9 & - & + & + & - & - & - \\
\hline 10 & - & + & - & + & - & - \\
\hline 11 & - & + & - & - & + & - \\
\hline 12 & - & + & - & - & - & - \\
\hline 13 & - & - & - & - & + & - \\
\hline 14 & - & - & - & - & - & + \\
\hline 15 & - & - & - & - & - & - \\
\hline
\end{tabular}

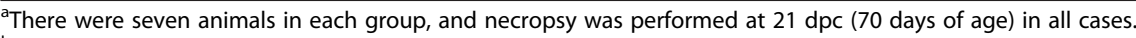

${ }^{b}$ Vaccine A: inactivated chimeric PCV1-2 vaccine (Fostera PCV, Zoetis, Madison, NJ, USA).

${ }^{\mathrm{C}}$ Vaccine B: experimental inactivated PCV2 vaccine. 
immunized with an experimental inactivated PCV2 vaccine administered intramuscularly as a $1.0 \mathrm{~mL}$ dose at 21 days of age.

At 49 days of age ( 0 days post challenge $(\mathrm{dpc}))$, the PCV2a-viremic pigs (groups 1, 2 and 5) and PCV2bviremic pigs (groups 7,8 , and 11 ) were challenged intranasally with $2 \mathrm{~mL}$ of PCV2b (strain SNUVR000463 (GenBank no. KF871068); $5^{\text {th }}$ passage; $1.0 \times 10^{5}$ tissue culture infective dose of $50 \%\left(\mathrm{TCID}_{50}\right) / \mathrm{mL}$ ) or PCV2a (strain SNUVR000032 (GenBank no. KF871067); $5^{\text {th }}$ passage; $1.0 \times 10^{5} \mathrm{TCID}_{50} / \mathrm{mL}$ ), respectively. The non-PCV2viremic pigs in groups 13 and 14 remained unvaccinated and were challenged with PCV2a or PCV2b at 49 days of age. The non-PCV2-viremic pigs in group 15 remained unvaccinated and unchallenged, and they served as the negative control group.

Blood was collected at $-42,-28,0,7,14$, and $21 \mathrm{dpc}$. For euthanasia, pigs were sedated by an intravenous injection of sodium pentobarbital and then euthanized by electrocution [14]. Superficial inguinal lymph nodes were collected for histopathology and in situ hybridization (ISH). Methods used in this study were approved by the Seoul National University Institutional Animal Care and Use Committee.

\section{Quantification of PCV DNA in blood}

DNA was extracted from serum samples using the QIAamp DNA mini kit. DNA extracts were used to quantify PCV2a and PCV2b genomic DNA copy numbers by real-time PCR as previously described [15].

\section{Serology}

IPMA and NA tests were performed using both a challenging PCV2a and PCV2b as previously described [16,17].

\section{Enzyme-linked immunospot (ELISPOT) assay}

The PCV2a and PCV2b antigens were prepared as previously described [8] and used as stimuli. The numbers of PCV2-specific interferon- $\gamma$-secreting cells (IFN- $\gamma$-SC) were determined in peripheral blood mononuclear cells (PBMC) as previously described [18,19].

\section{Histopathology and in situ hybridization}

For the morphometric analysis of histopathological changes in lymph nodes, three superficial inguinal lymph node sections were examined "blindly" as previously described [20]. Genotype-specific in situ hybridization (ISH) was used to detect PCV2a and PCV2b, respectively, in formalin-fixed, paraffin-embedded tissues [21]. Morphometric analysis of ISH was carried out as previously described [13].

\section{Statistical analysis}

Summary statistics were calculated for all groups to assess the overall quality of the data, including normality. The values of genomic copies of serological data and PCV2 viremia were transformed $\log _{2}$ and $\log _{10}$, respectively, prior to analysis. Continuous data (genomic copies of PCV2 DNA, serology and PCV2-specific IFN- $\gamma-S C$, number of in situ hybridization positive cells) were analyzed with a repeated measures analysis of variance (ANOVA). If the repeated measures ANOVA showed a significant effect, a one-way ANOVA with pairwise testing using the Tukey's adjustment was performed at each time point. If the distribution of variables was not normal, the non-parametric Kruskal-Wallis and MannWhitney tests were used to analyze the data. Discrete data (lymphoid lesion score) were analyzed by Chi-square and/ or the Fisher's exact test. A linear regression was performed to determine the correlation between PCV2 antibody titer at the day of vaccination (21 days of age) and the increment of PCV2 antibody titer at 28 days post vaccination (delta value, defined as PCV2 antibody titer at 28 days post vaccination minus PCV2 antibody titer at the day of vaccination).

\section{Results}

\section{PCV2 DNA in sera from PCV2a-viremic pigs}

The results of PCV2b DNA in the blood from PCV2aviremic pigs are summarized in Figure 1. PCV2a DNA was detected in the blood of PCV2a-viremic pigs throughout the experimental study. The PCV2a-viremic pigs that were vaccinated and then challenged with PCV2b (groups 1 and

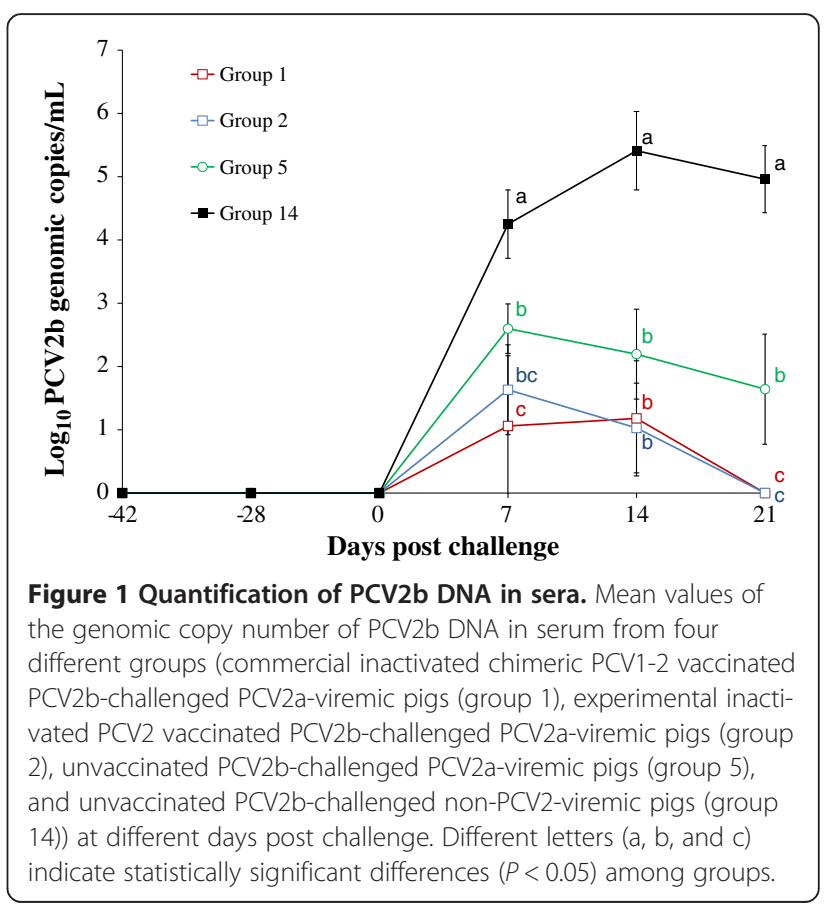


2) had a significantly $(P<0.05)$ lower number of genomic copies of PCV2b DNA in the blood compared to the unvaccinated PCV2b-challenged PCV2a-viremic pigs (group $5)$ and the unvaccinated PCV2b-challenged non-PCV2viremic pigs (group 14) at 7 and $21 \mathrm{dpc}$ (Figure 1). There was no significant difference in the $\log _{10}$ transformed genomic copy numbers (ranging from 3.27 to $4.12 \log _{10}$ PCV2a DNA copies $/ \mathrm{mL}$ ) of PCV2a DNA in PCV2a-viremic pigs regardless of PCV2 vaccination and challenge among the 6 groups (groups 1, 2, 3, 4, 5, and 6). No PCV2b was detected in the blood of unvaccinated unchallenged PCV2a-viremic (group 6) and non-PCV2-viremic negative control pigs (group 15).

\section{PCV2 DNA in sera from PCV2b-viremic pigs}

The results of PCV2a DNA in the blood from PCV2bviremic pigs are summarized in Figure 2. PCV2b DNA was detected in the blood of PCV2b-viremic pigs throughout the experimental study. The PCV2b-viremic pigs that were vaccinated and then challenged with PCV2a (groups 7 and $8)$ had a significantly $(P<0.05)$ lower number of genomic copies of PCV2a DNA in the blood than the unvaccinated PCV2a-challenged PCV2b-viremic pigs (group 11) and the unvaccinated PCV2a-challenged non-PCV2-viremic pigs (group 13) at 7, 14, and $21 \mathrm{dpc}$ (Figure 2). There was no significant difference in the $\log _{10}$ transformed genomic copy numbers (ranging from 3.11 to $4.32 \log _{10}$ PCV2b DNA copies $/ \mathrm{mL}$ ) of PCV2b DNA in PCV2b-viremic pigs regardless of PCV2 vaccination and challenge among 6 groups (groups 7, 8, 9, 10,11, and 12). PCV2a DNA was detected after $0 \mathrm{dpc}$ in the blood of pigs from PCV2a-

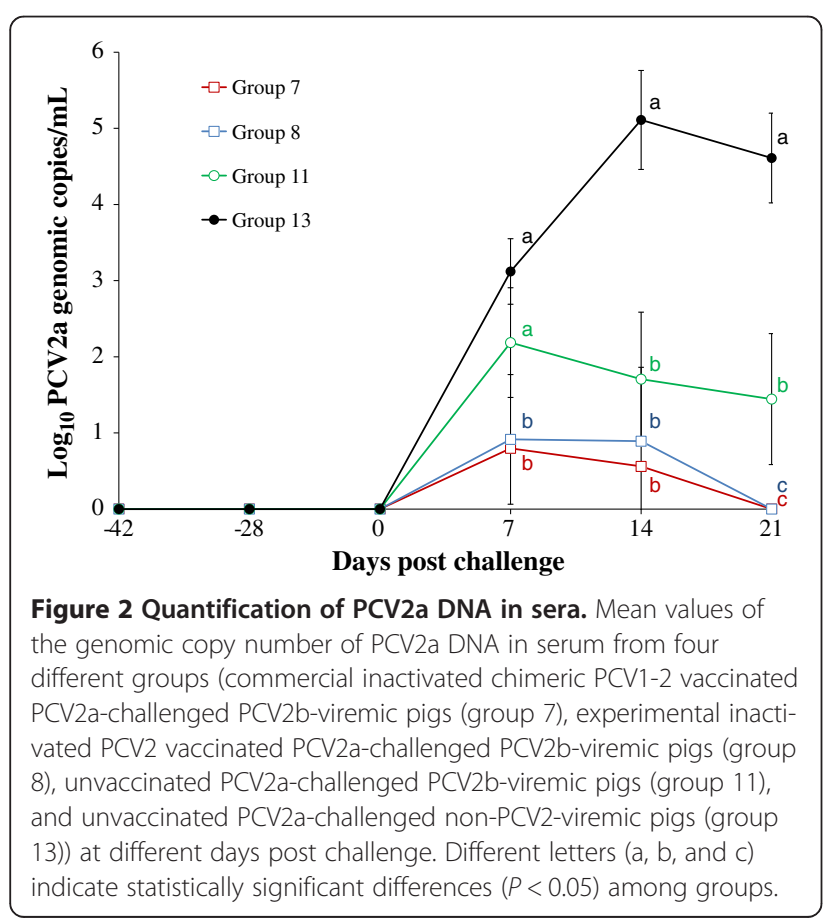

challenged groups $(7,8,11$, and 13) regardless of PCV2 vaccination. No PCV2a was detected in the blood of unvaccinated unchallenged PCV2b-viremic (group 12) and nonPCV2-viremic negative control pigs (group 15).

\section{Immunoperoxidase monolayer assay titers from PCV2a} (or 2b)-viremic pigs

The results of IPMA titers from PCV2a- and PCV2b viremic pigs are summarized in Figures $3 \mathrm{~A}$ and $4 \mathrm{~A}$, respectively. The vaccinated PCV2b-challenged PCV2aviremic pigs (groups 1 and 2$)$ had significantly $(P<0.05)$ higher PCV2-specific IPMA titers than the unvaccinated PCV2b-challenged PCV2a-viremic pigs (group 5) at 0, 7, and $14 \mathrm{dpc}$, and the unvaccinated unchallenged PCV2aviremic pigs (group 6) at $0,7,14$, and $21 \mathrm{dpc}$ (Figure 3A). The vaccinated $\mathrm{PCV} 2 \mathrm{a}$-challenged $\mathrm{PCV} 2 \mathrm{~b}$-viremic pigs (groups 7 and 8$)$ had significantly $(P<0.05)$ higher IPMA titers than the unvaccinated PCV2a-challenged PCV2bviremic pigs (group 11) at 0,7 , and $14 \mathrm{dpc}$, and the unvaccinated unchallenged PCV2b-viremic pigs (group 12) at 0 , 7, 14, and $21 \mathrm{dpc}$ (Figure 4A). No PCV2-specific IPMA titers were detected in unvaccinated unchallenged nonPCV2-viremic pigs (group 15).

\section{Neutralizing antibody titers from PCV2a (or 2b)-viremic pigs} The results of NA titers from PCV2a- and PCV2b-viremic pigs are summarized in Figures $3 \mathrm{~B}$ and $4 \mathrm{~B}$, respectively. The vaccinated PCV2b-challenged PCV2a-viremic pigs (groups 1 and 2$)$ had significantly $(P<0.05)$ higher PCV2specific NA titers than the unvaccinated PCV2b-challenged PCV2a-viremic pigs (group 5) at 0,7 , and $14 \mathrm{dpc}$ (Figure $3 \mathrm{~B})$. The vaccinated PCV2a-challenged PCV2bviremic pigs (groups 7 and 8 ) had significantly $(P<0.05)$ higher NA titers than the unvaccinated PCV2a-challenged PCV2b-viremic pigs (group 11) at $0,7,14$, and $21 \mathrm{dpc}$ (Figure 4B). No PCV2-specific NA titers were detected in unvaccinated unchallenged non-PCV2-viremic pigs (group 15).

\section{PCV2-specific interferon- $\gamma$-secreting cells from PCV2a (or 2b)-viremic pigs}

The results of PCV2-specific IFN- $\gamma$-SC from PCV2aand PCV2b-viremic pigs are summarized in Figures $3 \mathrm{C}$ and $4 \mathrm{C}$, respectively. The vaccinated $\mathrm{PCV} 2 \mathrm{~b}$-challenged PCV2a-viremic pigs (groups 1 and 2) had significantly $(P<0.05)$ higher mean numbers of PCV2-specific IFN- $\gamma$ $\mathrm{SC}$ than the unvaccinated PCV2b-challenged PCV2aviremic pigs (group 5) at 0,7 , and 14 dpc (Figure 3C). The vaccinated PCV2a-challenged PCV2b-viremic pigs (groups 7 and 8$)$ had significantly $(P<0.05)$ higher mean numbers of PCV2a- and PCV2b-specific IFN- $\gamma$-SC than the unvaccinated PCV2a-challenged PCV2b-viremic pigs (group 11) at 0, 7, and 14 dpc (Figure 4C). No PCV2- 
Figure 3 PCV2b-specific humoral and cell-mediated immune responses. $\log _{2}$ transformed group means for PCV2b-specific immunoperoxidase monolayer assay (IPMA) titers (A), neutralizing antibody (NA) titers (B), and frequency of PCV2b-specific interferon$\gamma$-secreting cells (IFN- $\gamma$-SC) $/ 10^{6}$ peripheral blood mononuclear cells (PBMC) (C) from 7 different groups (commercial inactivated chimeric PCV1-2 vaccinated PCV2b-challenged PCV2a-viremic pigs (group 1), experimental inactivated PCV2 vaccinated PCV2b-challenged PCV2aviremic pigs (group 2), commercial inactivated chimeric PCV1-2 vaccinated unchallenged PCV2a-viremic pigs (group 3), experimental inactivated PCV2 vaccinated unchallenged PCV2a-viremic pigs (group 4), unvaccinated PCV2b-challenged PCV2a-viremic pigs (group 5), unvaccinated unchallenged PCV2a-viremic pigs (group 6), and unvaccinated PCV2b-challenged non-PCV2-viremic pigs (group 14)). Different letters (a, b, c, and d) indicate statistically significant differences $(P<0.05)$ among groups.

specific IFN- $\gamma$-SC was observed in unvaccinated unchallenged non-PCV2-viremic pigs (group 15).

B

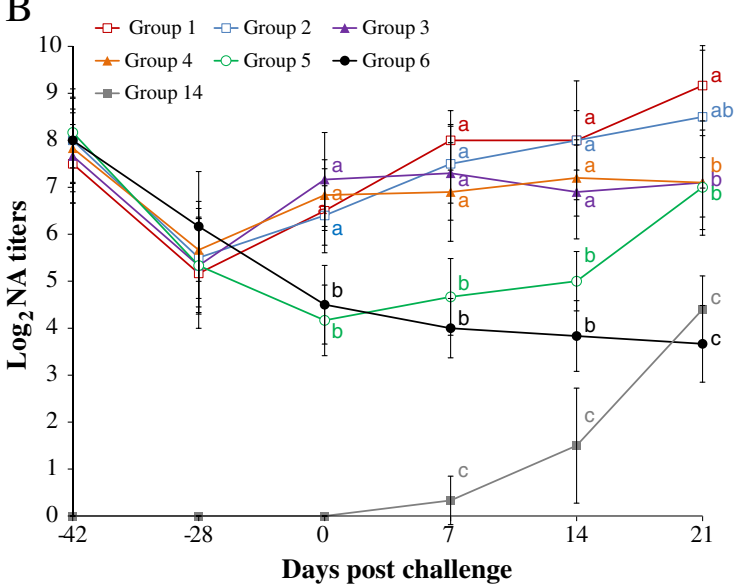

C

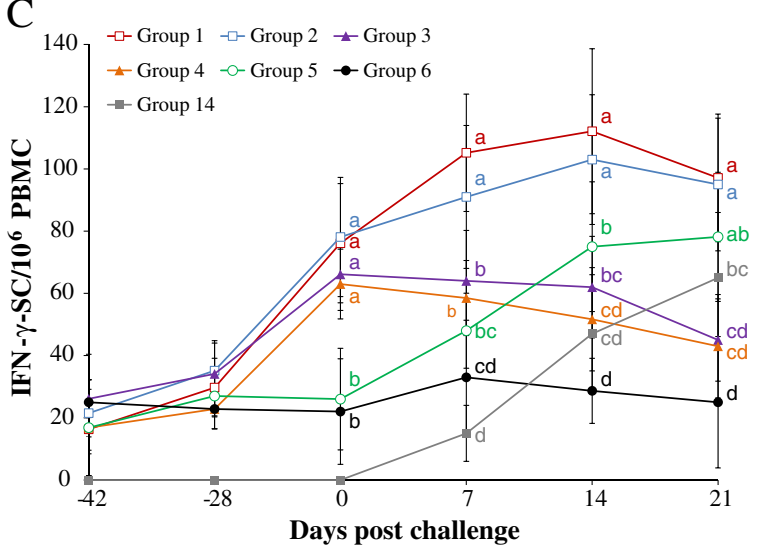

\section{Effects of maternally derived antibodies on PCV2 vaccine seroconversion}

The results show a significant negative correlation of humoral immune response with vaccination with a commercial inactivated chimeric PCV1-2 vaccine (Fostera PCV, Zoetis) in PCV2a- and PCV2b-viremic pigs $(\mathrm{r}=-0.8299, P=0.017$ for IPMA titers and $\mathrm{r}=-0.8467, P=0.012$ for NA titers) and vaccination with an experimental inactivated PCV2 vaccine in PCV2a- and PCV2b-viremic pigs $(\mathrm{r}=-0.7984, P=0.023$ for IPMA titers and $r=-0.8208, P=0.012$ for NA titers).

\section{Lymphoid lesion and in situ hybridization score from PCV2a (or 2b)-viremic pigs}

The results of lymphoid lesion and ISH score from PCV2a- and PCV2b-viremic pigs are summarized in Table 2. The pigs in six groups (group 1, 2, 3, 4, 5, and 6) had significantly $(P<0.05)$ lower lymphoid lesion scores and ISH PCV2b scores than the unvaccinated PCV2b-challenged non-PCV2-viremic pigs (group 14). The vaccinated PCV2a-challenged PCV2b-viremic pigs (groups 7 and 8 ) had significantly $(P<0.05)$ lower ISH PCV2a scores than the unvaccinated PCV2a-challenged PCV2b-viremic pigs (group 11). No hybridization signals for PCV2a and PCV2b were detected in lymph nodes from unvaccinated unchallenged non-PCV2-viremic pigs (group 15).

\section{Discussion}

The use of vaccination to immunize and protect pigs against PCV2 infection has been widely evaluated under experimental and field conditions [10,18,22-26]. Numerous experiments have shown that PCV2 vaccines are highly efficacious at protecting pigs against PCV2 infection. Ideally, PCV2 vaccination should be administered while residual maternally derived antibodies (MDA) are 
Figure 4 PCV2a-specific humoral and cell-mediated immune responses. $\log _{2}$ transformed group means for PCV2a-specific immunoperoxidase monolayer assay (IPMA) titers (A), neutralizing antibody (NA) titers (B), and frequency of PCV2a-specific interferon$\gamma$-secreting cells (IFN- $\gamma$-SC)/10 $10^{6}$ peripheral blood mononuclear cells (PBMC) (C) from 7 different groups (commercial inactivated chimeric PCV1-2 vaccinated PCV2a-challenged PCV2b-viremic pigs (group 7), experimental inactivated PCV2 vaccinated PCV2a-challenged PCV2bviremic pigs (group 8), commercial inactivated chimeric PCV1-2 vaccinated unchallenged PCV2b-viremic pigs (group 9), experimental inactivated $\mathrm{PCV} 2$ vaccinated unchallenged $\mathrm{PCV}$ 2b-viremic pigs (group 10), unvaccinated PCV2a-challenged PCV2b-viremic pigs (group 11), unvaccinated unchallenged PCV2b-viremic pigs (group 12), and unvaccinated PCV2a-challenged non-PCV2-viremic pigs (group 13)). Different letters ( $a, b, c$, and d) indicate statistically significant differences $(P<0.05)$ among groups.

minimal and before pigs become naturally infected. In the field, vaccination is likely administered routinely in the face of maternal immunity and infection in the field. Field data indicate that PCV2 vaccines are not inhibited by MDA when efficacy is assessed in terms of the reduction of PCV2-associated lesions and viral load in the serum $[23,25]$. Hence, MDA interference does not significantly hamper vaccine efficacy $[18,23,25]$. However, little scientific information is available describing the effect of PCV2 vaccination on PCV2-viremic and -seropositive pigs. The PCV2-viremic and -seropositive pigs used in this study had similar PCV2 viremia and serological profiles to naturally infected piglets $[5,12]$. Therefore, the experimental design attempted to mimic commercial swine rearing conditions to evaluate the response of PCV2viremic and -seropositive piglets after PCV2 vaccination and experimental PCV2 challenge.

In the present study, the vaccinated PCV2a-viremic pigs were challenged with PCV2b to differentiate initial PCV2 infection from challenging virus. PCV2 vaccines significantly reduced the amount of challenging PCV2a (or $2 \mathrm{~b}$ ) in the blood of PCV2b (or 2a)-viremic pigs that received a PCV2 vaccine and were subsequently challenged. The reduction of PCV2 viremia by the vaccine plays a critical role in controlling PCV2 infection [4]. A high PCV2 viremia has already been shown to be associated with the development of PCVAD [27-29]. Therefore, increased PCV2 viremia equates to an increased risk of developing PCVAD. The results of the present study demonstrate that PCV2 vaccination can protect PCV2-viremic pigs from a subsequent PCV2 challenge.

Despite the significant reduction of the challenging PCV2 in the blood as a result of PCV2 vaccination, PCV2a (or 2b)-viremic pigs that received a PCV2 vaccine followed by a PCV2b (or 2a) challenge retained a PCV2a (or 2b)-viremia as a result of previous exposure until the termination of the experimental study at 21 dpc. The failure to reduce or eliminate previously 
Table 2 Results of lymphoid lesion score and the number of in situ hybridization (ISH) positive cells for PCV2a and PCV2b at $21 \mathrm{dpc}$

\begin{tabular}{|c|c|c|c|c|}
\hline \multirow{2}{*}{$\begin{array}{l}\text { Challenge } \\
\text { status }\end{array}$} & \multirow[t]{2}{*}{ Group } & \multirow{2}{*}{$\begin{array}{l}\text { Lymphoid } \\
\text { lesion } \\
\text { score }\end{array}$} & \multicolumn{2}{|c|}{ In situ hybridization score } \\
\hline & & & PCV2a & PCV2b \\
\hline \multirow[t]{4}{*}{$2 a$} & 7 & $0.47 \pm 0.35^{a, b}$ & $0.71 \pm 1.25^{b}$ & $10.42 \pm 5.22^{b}$ \\
\hline & 8 & $0.51 \pm 0.42^{a, b}$ & $0.85 \pm 1.46^{b}$ & $11.14 \pm 5.89^{b}$ \\
\hline & 11 & $0.71 \pm 0.69^{a, b}$ & $8.14 \pm 6.06^{a}$ & $10.7 \pm 5.93^{b}$ \\
\hline & 13 & $1.57 \pm 0.53^{c}$ & $30.71 \pm 10.96^{c}$ & $0.0 \pm 0.0^{\mathrm{a}}$ \\
\hline \multirow[t]{4}{*}{$2 b$} & 1 & $0.53 \pm 0.52^{a, b}$ & $9.14 \pm 6.56^{\mathrm{a}}$ & $2.0 \pm 2.51^{\mathrm{a}}$ \\
\hline & 2 & $0.43 \pm 0.53^{\mathrm{a}, \mathrm{b}}$ & $7.28 \pm 6.87^{\mathrm{a}}$ & $2.42 \pm 3.1^{a}$ \\
\hline & 5 & $0.86 \pm 0.48^{b}$ & $8.42 \pm 5.15^{\mathrm{a}}$ & $7.14 \pm 4.14^{b}$ \\
\hline & 14 & $2.00 \pm 0.81^{c}$ & $0.0 \pm 0.0^{b}$ & $37.29 \pm 8.96^{c}$ \\
\hline \multirow[t]{7}{*}{ Non-challenged } & 3 & $0.29 \pm 0.49^{a}$ & $5.71 \pm 6.23^{\mathrm{a}}$ & $0.0 \pm 0.0^{a}$ \\
\hline & 4 & $0.23 \pm 0.41^{a}$ & $6.14 \pm 5.49^{a}$ & $0.0 \pm 0.0^{\mathrm{a}}$ \\
\hline & 6 & $0.31 \pm 0.37^{\mathrm{a}}$ & $8.57 \pm 6.07^{\mathrm{a}}$ & $0.0 \pm 0.0^{\mathrm{a}}$ \\
\hline & 9 & $0.35 \pm 0.39^{a, b}$ & $0.0 \pm 0.0^{b}$ & $8.85 \pm 5.17^{b}$ \\
\hline & 10 & $0.41 \pm 0.38^{a, b}$ & $0.0 \pm 0.0^{b}$ & $7.14 \pm 4.77^{b}$ \\
\hline & 12 & $0.43 \pm 0.49^{a, b}$ & $0.0 \pm 0.0^{b}$ & $9.14 \pm 5.27^{b}$ \\
\hline & 15 & $0.0 \pm 0.0^{\mathrm{a}}$ & $0.0 \pm 0.0^{b}$ & $0.0 \pm 0.0^{a}$ \\
\hline
\end{tabular}

Different letters $(a, b$, and $c)$ indicate significant $(P<0.05)$ difference among groups.

encountered PCV2 in the blood may result in a prolonged PCV2 viremia. It has been reported that PCV2 can maintain viremia up to $140 \mathrm{dpc}$ for PCV2a and 69 $\mathrm{dpc}$ for PCV2b $[30,31]$. The prolonged previously encountered PCV2 viremia may be transmitted to susceptible animals following direct contact with pigs and can perpetuate the infection within a herd. Further study is needed to determine the effect of previous exposure to PCV2 after PCV2 vaccination.

The efficacy of PCV2 vaccination in PCV2-viremic piglets can be affected by the presence of MDA. Therefore, the PCV2-viremic piglets may face potential interference from PCV2 antibodies present at the time of vaccination. Whether the efficacy of vaccination in piglets can be affected by the presence of MDA is controversial. Piglets with high IPMA titers $\left(>10 \log _{2}\right)$ have been shown to experience interference with the development of a humoral immune response following vaccination, while piglets with moderate titers $(<8$ $\log _{2}$ ) do not [22]. In the present study, PCV2-viremic piglets had moderate IPMA $\left(<8 \log _{2}\right)$ and NA $(<7$ $\left.\log _{2}\right)$ titers at the time of vaccination. Moreover, the PCV2 vaccine elicited a high level of IPMA and NA titers, and the frequency of IFN- $\gamma$-SC in PCV2-viremic and -seropositive piglets was high even in the presence of MDA. These two immunological parameters are responsible for the reduction of PCV2 viremia and control PCV2 infection [28,32]. These data suggest that preexisting, moderate levels of MDA do not interfere with the development of an active immune response after PCV2 vaccination in PCV2-viremic piglets.

There were no significant differences in the frequency of PCV2-specific IFN- $\gamma$-SC in response to the whole virus when either PCV2a or PCV2b was used as a recall antigen, as demonstrated in a previous study [33]. The PCV2a-based commercial vaccine and the PCV2b-based experimental vaccine induced similar profiles of PCV2aand PCV2b-specific IFN- $\gamma$-SC in the PCV2a- and PCV2bviremic pigs when either PCV2a or PCV2b was used as a recall antigen. These results suggest the existence of conserved immunodominant T-cell epitopes between both genotypes, which is further supported by the cross-protection of PCV2a-based vaccines against a PCV2b challenge $[34,35]$. These results are clinically significant because all the commercial PCV2 vaccines currently used worldwide are based on the PCV2a genotype.

Rather than PCV2 vaccination, the reduction of PCV2b (or 2a) viremia in pigs previously exposed to PCV2a (or 2b) may be due to cross protection between the two genotypes. PCV2a- (or 2b-) viremic pigs that were challenged with PCV2b (or 2a) had significantly reduced levels of challenging PCV2 in the blood. These results were in agreement with a previous study [36]. Although cross protection between PCV2 and PCV2b exists [36], PCV2aviremic pigs that received a PCV2 vaccine followed by a PCV2b challenge had a significantly lower number of genomic copies of challenging PCV2b in the serum than the unvaccinated PCV2a-viremic pigs that were only challenged with PCV2b. These results indicate that active immune responses by PCV2 vaccination, rather than natural infection or passively transferred MDA, play an important role in reducing PCV2b viremia in PCV2a-viremic pigs that received a PCV2 vaccine followed by a PCV2b challenge and vice versa.

PCV2-associated microscopic lesions were not prominent in this study because the pigs were challenged with PCV2 alone rather than co-challenged with other viruses, such as PRRSV or PPV. Vaccination effectively reduced the number of PCV2-associated microscopic lesions and the level of PCV2 DNA in the lymphoid tissues of PCV2-viremic piglets that received a PCV2 vaccine followed by a PCV2 challenge. Many pig producers may believe that PCV2-viremic or -seropositive piglets contribute to vaccine failure. The results from this study demonstrate that the PCV2 vaccine was effective in reducing PCV2 viremia and lymphoid PCV2 DNA even when used on PCV2-viremic pigs with passively acquired MDA at the time of vaccination. 


\section{Competing interests}

The authors declare that they have no competing interests.

\section{Authors' contributions}

HWS performance of the experimental trials, data analysis and writing of the manuscript, CP and $\mathrm{KH}$ preparation of the inoculum and lab analysis and inoculation of the virus, CC development of the protocol, design of the study, review of the final manuscript, approval for publication. All authors read and approved the final manuscript.

\section{Acknowledgements}

This research was supported by Technology Development Program for Agriculture and Forestry, Ministry for Food, Agriculture, Forestry and Fisheries, Republic of Korea. This research was also supported by contract research funds of the Research Institute for Veterinary Science (RIVS) from the College of Veterinary Medicine and by Brain Korea 21 Plus Program for Creative Veterinary Science Research in the Republic of Korea.

Received: 3 July 2013 Accepted: 21 January 2014

Published: 2 February 2014

\section{References}

1. Meehan BM, McNeilly F, Todd D, Kennedy S, Jewhurst VA, Ellis JA, Hassard LE, Clark EG, Haines DM, Allan GM: Characterization of novel circovirus DNAs associated with wasting syndromes in pigs. J Gen Virol 1998, 79:2171-2179

2. Chae C: Postweaning multisystemic wasting syndrome: a review of aetiology, diagnosis and pathology. Vet J 2004, 168:41-49.

3. Chae C: A review of porcine circovirus 2-associated syndromes and diseases. Vet J 2005, 169:326-336.

4. Chae C: Commercial porcine circovirus type 2 vaccines: Efficacy and clinical application. Vet J 2012, 194:151-157

5. Shen $H$, Wang C, Madson DM, Opriessnig T: High prevalence of porcine circovirus viremia in newborn piglets in five clinically normal swine breeding herds in North American. Prev Vet Med 2010, 97:228-236.

6. Chae C: Porcine circovirus type 2 and its associated disease in Korea. Virus Res 2012, 164:107-113.

7. Ha Y, Shin JH, Chae C: Colostral transmission of porcine circovirus 2 (PCV-2): reproduction of post-weaning multisystemic wasting syndrome in pigs fed milk from PCV-2-infected sows with post-natal porcine parvovirus infection or immunostimulation. J Gen Virol 2010, 91:1601-1608.

8. Calsamiglia M, Fraile L, Espinal A, Cuxart A, Seminati C, Martin M, Mateu E, Domingo M, Segalés J: Sow porcine circovirus type 2 (PCV2) status effect on litter mortality in postweaning multisystemic wasting syndrome (PMWS). Res Vet Sci 2007, 82:299-304.

9. O'Neill KC, Hemann M, Gimenez-Lirola LG, Halbur PG, Opriessnig T: Vaccination of sows reduces the prevalence of PCV-2 viraemia in their piglets under field conditions. Vet Rec 2012, 171:425

10. Opriessnig T, Patterson AR, Madson DM, Pal N, Ramamoorthy S, Meng XJ, Halbur PG: Comparison of the effectiveness of passive (dam) versus active (piglet) immunization against porcine circovirus type 2 (PCV2) and impact of passively derived PCV2 vaccine-induced immunity on vaccination. Vet Microbiol 2010, 142:177-183.

11. Shen HG, Beach NM, Huang YW, Halbur PG, Meng XJ, Opriessnig T: Comparison of commercial and experimental porcine circovirus type 2 (PCV2) vaccines using a triple challenge with PCV2, porcine reproductive and respiratory syndrome virus (PRRSV), and porcine parvovirus (PPV). Vaccine 2010, 28:5960-5966.

12. Gerber PF, Garrocho FM, Lana AMQ, Lobato ZIP: Fetal infections and antibody profiles in pigs naturally infected with porcine circovirus type 2 (PCV2). Can J Vet Res 2012, 76:38-44.

13. Kim D, Kim CH, Han K, Seo HW, Oh Y, Park C, Kang I, Chae C: Comparative efficacy of commercial Mycoplasma hyopneumoniae and porcine circovirus 2 (PCV2) vaccines in pigs experimentally infected with $M$. hyopneumoniae and PCV2. Vaccine 2011, 29:3206-3212.

14. Beaver BV, Reed W, Leary S, McKiernan B, Bain F, Schultz R, Bennett BT, Pascoe P, Shull E, Cork LC, Francis-Floyd R, Amass KD, Johnson R, Schmidt $\mathrm{RH}$, Underwood W, Thornton GW, Kohn B: 2000 report of the AVMA panel on euthanasia. J Am Vet Med Assoc 2001, 218:669-696.

15. Gagnon CA, Del-Castillo JR, Music N, Fontaine G, Harel J, Tremblay D: Development and use of a multiplex real-time quantitative polymerase chain reaction assay for detection and differentiation of Porcine circovirus-2 genotypes 2a and 2b in an epidemiological survey. J Vet Diagn Invest 2008, 20:545-558.

16. Fort M, Olvera A, Sibila M, Segalés J, Mateu E: Detection of neutralizing antibodies in postweaning multisystemic wasting syndrome (PMWS)affected and non-PMWS-affected pigs. Vet Microbiol 2007, 125:244-255.

17. Pogranichnyy RM, Yoon KJ, Harms PA, Swenson SL, Zimmerman JJ, Sorden SD: Characterization of immune response of young pigs to porcine circovirus type 2 infection. Viral Immunol 2000, 13:143-153.

18. Seo HW, Han K, Oh Y, Park C, Chae C: Efficacy of a reformulated inactivated chimeric PCV1-2 vaccine based on clinical, virological, pathological and immunological examination under field conditions. Vaccine 2012, 30:6671-6677.

19. Diaz I, Mateu E: Use of ELISPOT and ELISA to evaluate IFN- $\gamma$, IL-10 and IL-4 responses in conventional pigs. Vet Immunol Immunopathol 2005, 106:107-112.

20. Kim J, Chae C: Expression of monocyte chemoattractant protein-1 and macrophage inflammatory protein-1 in porcine circovirus 2-induced granulomatous inflammation. J Comp Pathol 2004, 131:121-126.

21. Kim D, Ha Y, Oh Y, Han K, Noh SH, Kim C, Kim S-H, Chae C: Development of in situ hybridization assay that differentiates between two genotypes of Porcine circovirus-2 in formalin-fixed, paraffin-embedded tissues. $J$ Vet Diagn Invest 2010, 22:231-233.

22. Fort M, Sibila M, Perez-Martin E, Nofrarias M, Mateu E, Segalés J: One dose of a porcine circovirus 2 (PCV2) sub-unit vaccine administered to 3week-old conventional piglets elicits cell-mediated immunity and significantly reduces PCV2 viremia in an experimental model. Vaccine 2009, 27:4031-4037.

23. Fraile L, Grau-Roma L, Sarasola P, Sinovas N, Nofrarias M, Lopez-Jimenez R, Lopez-Soria S, Silbila M, Segalés J: Inactivated PCV2 one shot vaccine applied in 3-week-old piglets: Improvement of production parameters and interaction with maternally derived immunity. Vaccine 2012, 30:1986-1992.

24. Kixmoller M, Ritzmann M, Eddicks M, Saalmuller A, Elbers K, Fachinger V: Reduction of PMWS-associated clinical signs and co-infections by vaccination against PCV2. Vaccine 2008, 26:3443-3451.

25. Martelli $P$, Ferrari $L$, Morganti $M$, Angelis DE, Bonilauri $P$, Guazzetti $S$, Caleffi A, Borghetti P: One dose of a porcine circovirus 2 subunit vaccine induces humoral and cell-mediated immunity and protects against porcine circovirus-associated disease under field conditions. Vet Microbiol 2011, 149:339-351.

26. Opriessnig T, Patterson AR, Madson DM, Pal N, Halbur PG: Comparison of efficacy of commercial one dose and two dose PCV2 vaccines using a mixed PRRSV-PCV2-SIV clinical infection model 2-3-months post vaccination. Vaccine 2009, 27:1002-1007.

27. Olvera A, Sibila M, Calsamiglia M, Segalés J, Domingo M: Comparison of porcine circovirus type 2 load in serum quantified by a real time PCR in postweaning multisystemic wasting syndrome and porcine dermatitis and nephropathy syndrome naturally affected pigs. J Virol Methods 2004, 117:75-80.

28. Meerts P, Misinzo G, Lefebvre D, Nielsen J, Botner A, Kristensen CS, Nauwynck H: Correlation between the presence of neutralizing antibodies against porcine circovirus 2 (PCV2) and protection against replication of the virus and development of PCV2-associated disease. BMC Vet Res 2006, 2:6.

29. Liu Q, Wang L, Willson P, Babiuk A: Quantitative, competitive PCR analysis of porcine circovirus DNA in serum from pigs with postweaning multisystemic wasting syndrome. J Clin Microbiol 2000, 38:3474-3477.

30. Opriessnig T, Prickett JR, Madon DM, Shen H-G, Juhan NM, Pogranichniy RM, Meng X-J, Halbur PG: Porcine circovirus type 2 (PCV2)-infection and reinoculation with homologous or heterologous strains: virological, serological, pathological and clinical effects in growing pigs. Vet Res 2010, 41:31.

31. Patterson AR, Ramamoorthy S, Madson DM, Meng XJ, Halbur PG, Opriessnig $\mathrm{T}$ : Shedding and infection dynamics of porcine circovirus type 2 (PCV2) after experimental infection. Vet Microbiol 2011, 149:91-98.

32. Meerts P, Van-Gucht S, Cox E, Vandebosch A, Nauwynck HJ: Correlation between type of adaptive immune response against porcine circovirus type 2 and level of virus replication. Viral Immunol 2005, 18:333-341.

33. Fort $M$, Sibila $M$, Nofrarias $M$, Perez-Martin E, Olvera A, Mateu E, Segalés J: Porcine circovirus type 2 (PCV2) Cap and Rep protein are involved in the development of cell-mediated immunity upon PCV2 infection. Vet Immunol Immunopathol 2010, 137:226-234. 
34. Fort M, Sibila M, Allepuz A, Mateu E, Roerink F, Segalés J: Porcine circovirus type 2 (PCV2) vaccination of conventional pigs prevents viremia against PCV2 isolates of different genotypes and geographic origins. Vaccine 2008, 26:1063-1071.

35. Seo HW, Oh Y, Han K, Park C, Chae C: Reduction of porcine circovirus type 2 (PCV2) viremia by a reformulated inactivated chimeric PCV1-2 vaccineinduced humoral and cellular immunity after experimental PCV2 challenge. BMC Vet Res 2012, 8:194

36. Opriessnig T, Ramamoorthy S, Madson DM, Patterson AR, Pal N, Carman S, Meng XJ, Halbur PG: Differences in virulence among porcine circovirus type 2 isolates are unrelated to cluster type $2 \mathrm{a}$ or $2 \mathrm{~b}$ and prior infection provides heterologous protection. J Gen Virol 2008, 89:2482-2491.

doi:10.1186/1297-9716-45-13

Cite this article as: Seo et al:: Effect of porcine circovirus type 2 (PCV2) vaccination on PCV2-viremic piglets after experimental PCV2 challenge. Veterinary Research 2014 45:13.

\section{Submit your next manuscript to BioMed Central and take full advantage of:}

- Convenient online submission

- Thorough peer review

- No space constraints or color figure charges

- Immediate publication on acceptance

- Inclusion in PubMed, CAS, Scopus and Google Scholar

- Research which is freely available for redistribution 